\title{
On the Number of Bins in Equilibria for Signaling Games
}

\author{
Serkan Sarıtaş*, Philippe Furrer ${ }^{\dagger}$, Sinan Gezici ${ }^{\ddagger}$, Tamás Linder ${ }^{\S}$, and Serdar Yüksel ${ }^{\S}$ \\ *Division of Decision and Control Systems, KTH Royal Institute of Technology, SE-10044, Stockholm, Sweden, saritas@kth.se \\ †Oliver Wyman Inc., M5J 0A1, Toronto, Ontario, Canada, phil.furrer@oliverwyman.com \\ $\ddagger$ Dept. of Electrical and Electronics Engineering, Bilkent University, 06800, Ankara, Turkey, gezici@ee.bilkent.edu.tr

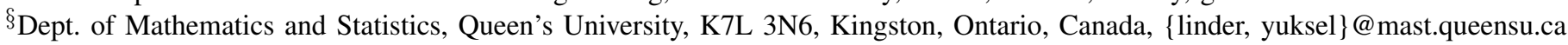

\begin{abstract}
We investigate the equilibrium behavior for the decentralized quadratic cheap talk problem in which an encoder and a decoder, viewed as two decision makers, have misaligned objective functions. In prior work, we have shown that the number of bins under any equilibrium has to be at most countable, generalizing a classical result due to Crawford and Sobel who considered sources with density supported on $[0,1]$. In this paper, we refine this result in the context of exponential and Gaussian sources. For exponential sources, a relation between the upper bound on the number of bins and the misalignment in the objective functions is derived, the equilibrium costs are compared, and it is shown that there also exist equilibria with infinitely many bins under certain parametric assumptions. For Gaussian sources, it is shown that there exist equilibria with infinitely many bins.
\end{abstract}

\section{INTRODUCTION}

Signaling games and cheap talk are concerned with a class of Bayesian games where a privately informed player (encoder or sender) transmits information (signal) to another player (decoder or receiver), who knows the probability distribution of the possible realizations of the private information of the encoder. In these games/problems, the objective functions of the players are not aligned, unlike in classical communication problems. The cheap talk problem was introduced in the economics literature by Crawford and Sobel [1], who obtained the striking result that under some technical conditions on the cost functions, the cheap talk problem only admits equilibria that involve quantized encoding policies. This is in significant contrast to the usual communication/information theoretic case where the objective functions are aligned. Therefore, as indicated in [1], the similarity of players' interests (objective functions) indicates how much and to which extent the information should be revealed by the encoder; namely, the message about the private information should be strategically designed and transmitted by the encoder. In this paper, we discuss extensions and generalizations of strategic information transmission and cheap talk by focusing on exponential and Gaussian distributions (rather than sources with a density supported on $[0,1]$ as studied in [1]), and characterize the equilibrium solutions and properties for these kind of sources.

\section{A. Problem Definition}

The focus of this paper is to address the following problems:
1) Number of Bins: In our previous work [2], we showed that, since the distances between the optimal decoder actions are lower bounded by [2, Theorem 3.2], the quantized nature of an equilibrium holds for arbitrary scalar sources, rather than only for sources with a density supported on $[0,1]$ as studied in the seminal paper by Crawford and Sobel [1]. Hence, for bounded sources, it can easily be deduced that the number of bins at the equilibrium must be bounded. For example, for a uniform source on $[0,1]$ and quadratic objective functions, [1] provides an upper bound on the number of quantization bins as a function of the bias $b$. Accordingly, for unbounded sources, the following problems are of interest:

- For unbounded sources, either one-sided or two-sided, is there an upper bound on the number of bins at the equilibrium as a function of bias $b$ ? As a special case, is it possible to have only a non-informative equilibrium; i.e., the upper bound on the number of bins is one?

- Does there exist an equilibrium with infinitely many bins?

At this point, one can ask why bounding the number of bins is important. Finding such bound is useful since if one can show that there only exists a finite number of bins, and if for every bin there is a finite number of distinct equilibria, then the total number of equilibria would be finite; this will allow for a feasible setting where the decision makers can coordinate their policies.

Furthermore, in a recent work, where we generalized signaling games and cheap talk problems to dynamic (multistage) setups, a crucial property that allowed the generalization was the assumption that the number of bins for each stage equilibrium, conditioned on the past actions, is uniformly bounded [3, Theorem 2.4]. In view of this, showing that the number of bins is finite would be a useful technical result.

2) Equilibrium Selection: Attaining the upper bound $N$ on the number of bins at the equilibrium implies that there exists at least one equilibrium with $1,2, \ldots N$ bins due to [1, Theorem 1], and thus, a new question arises: among these multiple equilibria, which one is preferred by the players? Results in [1] show that an equilibrium with more bins is preferable for both the encoder and the decoder for any source with a density bounded on $[0,1]$. Accordingly, for more general sources, one can ask that 
- if there exists more than one equilibrium, which one of these should be selected by the encoder and the decoder?

- to what extent can one argue that more bins lead to better performance?

Indeed, it is important to know whether in general a higher number of bins implies more desirable equilibria. If such a monotonic behavior holds for a class of sources, then both players will prefer to have an equilibrium with the highest number of bins.

\section{B. Preliminaries}

In cheap talk, there are two players with misaligned objective functions. An informed player (encoder) knows the value of an $\mathbb{M}$-valued random variable $M$ and transmits an $\mathbb{X}$ valued random variable $X$ to another player (decoder), who generates his $\mathbb{M}$-valued decision $U$ upon receiving $X$. The policies of the encoder and the decoder are assumed to be deterministic; i.e., $x=\gamma^{e}(m)$ and $u=\gamma^{d}(x)=\gamma^{d}\left(\gamma^{e}(m)\right)$. Let $c^{e}(m, u)$ and $c^{d}(m, u)$ denote the cost functions of the encoder and the decoder, respectively, when the action $u$ is taken for the corresponding message $m$. Then, given the encoding and decoding policies, the encoder's induced expected cost is $J^{e}\left(\gamma^{e}, \gamma^{d}\right)=\mathbb{E}\left[c^{e}(M, U)\right]$, whereas the decoder's induced expected cost is $J^{d}\left(\gamma^{e}, \gamma^{d}\right)=\mathbb{E}\left[c^{d}(M, U)\right]$. Here, we assume real valued random variables and quadratic cost functions; i.e., $\mathbb{M}=\mathbb{X}=\mathbb{R}, c^{e}(m, u)=(m-u-b)^{2}$ and $c^{d}(m, u)=(m-u)^{2}$, where $b$ denotes a bias term which is common knowledge between the players. We assume the simultaneous-move game; i.e., the encoder and the decoder announce their policies at the same time. Then a pair of policies $\left(\gamma^{*, e}, \gamma^{*, d}\right)$ is said to be a Nash equilibrium [4] if

$$
\begin{aligned}
& J^{e}\left(\gamma^{*, e}, \gamma^{*, d}\right) \leq J^{e}\left(\gamma^{e}, \gamma^{*, d}\right) \text { for all } \gamma^{e} \in \Gamma^{e}, \\
& J^{d}\left(\gamma^{*, e}, \gamma^{*, d}\right) \leq J^{d}\left(\gamma^{*, e}, \gamma^{d}\right) \text { for all } \gamma^{d} \in \Gamma^{d},
\end{aligned}
$$

where $\Gamma^{e}$ and $\Gamma^{d}$ are the sets of all deterministic (and Borel measurable) functions from $\mathbb{M}$ to $\mathbb{X}$ and from $\mathbb{X}$ to $\mathbb{M}$, respectively. As observed from the definition in (1), under the Nash equilibrium, each individual player chooses an optimal strategy given the strategy chosen by the other player.

Due to results obtained in [1] and [2], we know that the encoder policy consists of convex cells (bins) at a Nash equilibrium ${ }^{1}$. Now consider an equilibrium with $N$ bins, and let the $k$-th bin be the interval $\left[m_{k-1}, m_{k}\right)$ with $m_{0}<m_{1}<$ $\ldots<m_{N}$ and let $l_{k}$ denote the length of the $k$-th bin; i.e., $l_{k}=m_{k}-m_{k-1}$ for $k=1,2, \ldots, N$ (Note that $m_{0}=0$ and $m_{N}=+\infty$ for an exponential source, whereas $m_{0}=-\infty$ and $m_{N}=+\infty$ for a Gaussian source). By [2, Theorem 3.2],

\footnotetext{
${ }^{1}$ We note that, unlike Crawford and Sobel's simultaneous Nash equilibrium formulation, if one considers a Stackelberg formulation (see [4, p.133] for a definition), then the problem would reduce to a classical communication problem since the encoder would be committed a priori and the equilibrium would not be quantized; i.e., there exist affine equilibria [2], [3], [5]-[7]. Recently, the problem of strategical coordination has been considered in [8]; specifically, the information design and a point-to-point strategic sourcechannel coding problems (originated from the Bayesian persuasion game [9]) between an encoder and a decoder with non-aligned utility functions have been investigated under the Stackelberg equilibrium.
}

at the equilibrium, decoder's best response to encoder's action is characterized by

$$
u_{k}=\mathbb{E}\left[M \mid m_{k-1} \leq M<m_{k}\right]
$$

for the $k$-th bin; i.e., the optimal decoder action is the centroid for the corresponding bin. From encoder's point of view, the best response of the encoder to decoder's action is determined by the nearest neighbor condition [2, Theorem 3.2] as follows:

$$
u_{k+1}-m_{k}=\left(m_{k}-u_{k}\right)-2 b \Leftrightarrow m_{k}=\frac{u_{k}+u_{k+1}}{2}+b .
$$

Due to the definition of Nash equilibirum [4], these best responses in (2) and (3) must match each other, and only then the equilibrium can be characterized; i.e., for a given number of bins, the positions of the bin edges are chosen by the encoder, and the centroids are determined by the decoder. Alternatively, the problem can be considered as a quantization game in which the boundaries are determined by the encoder and the reconstruction values are determined by the decoder.

Note that at the equilibrium of this quantization game, the relation between the encoder cost and the decoder cost can be expressed as $J^{e}\left(\gamma^{*, e}, \gamma^{*, d}\right)=J^{d}\left(\gamma^{*, e}, \gamma^{*, d}\right)+b^{2}$. Since the difference between the encoder cost and the decoder cost is always $b^{2}$ regardless of the number of bins, the equilibrium preferences (i.e., which equilibrium to select) for the players are aligned under the quadratic cost assumption.

Based on the above, the problems we consider in this paper can be formulated more formally as follows:

1) Number of Bins: For a given finite (or infinite) $N$, does there exist an equilibrium; i.e., is it possible to find the optimal encoder actions (the boundaries of the bins) $m_{0}, m_{1}, \ldots, m_{N}$ and decoder actions (the centroids of the bins) $u_{1}, u_{2}, \ldots, u_{N}$ which satisfy (2) and (3) simultaneously? Here two possible different methods are:

(i) Lloyd's Method I: After the initial selection of $m_{0}, m_{1}, \ldots, m_{N}$, determine $u_{1}, u_{2}, \ldots, u_{N}$ by (2), and after updating $u_{1}, u_{2}, \ldots, u_{N}$, find the corresponding $m_{0}, m_{1}, \ldots, m_{N}$ by (3). Then, continue this iteration. For this approach, the convergence of this Lloyd-Max iteration is the key issue.

(ii) Fixed-point approach: By combining (2) and (3),

$$
m_{k}=\frac{\mathbb{E}\left[M \mid m_{k-1} \leq M<m_{k}\right]+\mathbb{E}\left[M \mid m_{k} \leq M<m_{k}+1\right]}{2}+b
$$

is obtained for $k=1,2, \ldots, N$. Then, the problem reduces to determining whether there exists a fixed vector $m_{0}, m_{1}, \ldots, m_{N}$ satisfying these equations.

2) Equilibrium Selection: Let $J^{d, N}$ denote the decoder cost at the equilibrium with $N$ bins. Then, is it true that $J^{d, N}>J^{d, N+1}$ for any finite $N$, or even, is $J^{d, N}>J^{d, \infty}$ if an equilibrium with infinitely many bins exists?

\section{Related Literature}

Cheap talk and signaling game problems find applications in networked control systems when a communication channel/network is present among competitive and non-cooperative decision makers [4], [10]. Also, there have been a number 
of related results in the economics and control literature in addition to the seminal work by Crawford and Sobel, which are reviewed in [2], [3] (see [11] for an extensive survey).

The quantized nature of the equilibrium makes game theory connected with the quantization theory. For a comprehensive survey regarding the history of quantization and results on the optimality and convergence properties of different quantization techniques (including Lloyd's methods), we refer to [12]. In particular, [13] shows that, for sources with a log-concave density, Lloyd's Method I converges to the unique optimal quantizer. It was shown in [14] and [15] that Lloyd's Method I converges to the globally optimal quantizer if the source density is continuous and log-concave, and if the error weighting function is convex and symmetric. For sources with bounded support, the condition on the source was relaxed to include all continuous and positive densities in [16], and convergence of Lloyd's Method I to a (possibly) locally optimal quantizer was proved. The number of bins of an optimal entropyconstrained quantizer is investigated in [17], and conditions under which the number of bins is finite or infinite are presented. As an application to smart grids, [18] considers the design of signaling schemes between a consumer and an electricity aggregator with finitely many messages (signals); the best responses are characterized and the maximum number of messages (i.e., quantization bins) are found using Lloyd's Method II via simulation.

The existence of multiple quantized equilibria necessitates a theory to specify which equilibrium point is the solution of a given game. Two different approaches are taken to achieve a unique equilibrium. One of them reduces the multiplicity of equilibria by requiring that off-the-equilibrium-path beliefs satisfy an additional restriction (e.g., by shrinking the set of players' rational choices) [19], [11]. As introduced in [20], the other approach presents a theory that selects a unique equilibrium point for each finite game as its solution; i.e., one and only one equilibrium points out of the set of all equilibrium points of this kind (e.g., see [21] for the application).

\section{Contributions}

(i) Under the exponential source assumption with a negative bias; i.e., $b<0$, we obtain an upper bound on the number of bins at the equilibrium and show that the equilibrium cost reduces as the number of bins increases.

(ii) Under the exponential source assumption with a positive bias; i.e., $b>0$, we prove that there exists a unique equilibrium with $N$ bins for any $N \in \mathbb{N}$ and there is no upper bound on the number of bins; in fact, there exist equilibria with infinitely many bins. Further, the equilibrium cost achieves its minimum at the equilibrium with infinitely many bins.

(iii) Under the Gaussian source assumption, we show that there always exist equilibria with infinitely many bins regardless of the value of $b$.

Due to space constraints, here we provide only the proof sketches; the detailed proofs are available in [22].

\section{EXPONENTIAL DistribUtion}

In this section, the source is assumed to be exponential and the number of bins at the equilibria is investigated. Before delving into the technical results, we observe the following fact:

Fact 2.1: Let $M$ be an exponentially distributed r.v. with a positive parameter $\lambda$ : i.e., the probability distribution function (PDF) of $M$ is $\mathrm{f}(m)=\lambda \mathrm{e}^{-\lambda m}$ for $m \geq 0$. The expectation and the variance of an exponential r.v. truncated to the interval $[a, b]$ are $\mathbb{E}[M \mid a<M<b]=\frac{1}{\lambda}+a-\frac{b-a}{\mathrm{e}^{\lambda(b-a)}-1}$ and $\operatorname{Var}(M \mid a<M<b)=\frac{1}{\lambda^{2}}-\frac{(b-a)^{2}}{\mathrm{e}^{\lambda(b-a)}+\mathrm{e}^{-\lambda(b-a)}-2}$, respectively.

The following result shows the existence of an equilibrium with finitely many bins. Here, $\lfloor x\rfloor$ denotes the largest integer less than or equal to $x$.

Proposition 2.1: Suppose $M$ is exponentially distributed with parameter $\lambda$. Then, for $b<0$, any Nash equilibrium has at most $\left\lfloor-\frac{1}{2 b \lambda}+1\right\rfloor$ bins with monotonically increasing binlengths.

Proof: Since $u_{N}=\mathbb{E}\left[M \mid m_{N-1} \leq M \leq m_{N}=\infty\right]=$ $m_{N-1}+\frac{1}{\lambda}$, it follows that

$$
\begin{aligned}
& \frac{1}{\lambda}=u_{N}-m_{N-1}=\left(m_{N-1}-u_{N-1}\right)-2 b \\
& \quad>\left(u_{N-1}-m_{N-2}\right)-2 b=\left(m_{N-2}-u_{N-2}\right)-2(2 b) \\
& \quad \vdots \\
& \quad>u_{1}-m_{0}-(N-1)(2 b)>-(N-1)(2 b) .
\end{aligned}
$$

Here, the inequalities follow from the fact that the exponential PDF is monotonically decreasing, which implies that binlengths are monotonically increasing; i.e., $l_{k}>l_{k-1}$. Thus, for $b<0, N \leq\left\lfloor-\frac{1}{2 b \lambda}+1\right\rfloor$ is obtained.

This is an important result as it provides us with a closed form expression for the maximum bit rate required by a certain system to operate at a steady state. For example, there can be at most one bin at the equilibrium (i.e., a non-informative equilibrium) if $N \leq\left\lfloor-\frac{1}{2 b \lambda}+1\right\rfloor<2 \Leftrightarrow-\frac{1}{2 b \lambda}<1 \Leftrightarrow b<-\frac{1}{2 \lambda}$. However, this result does not characterize the equilibrium completely; i.e., it does not give a condition on the existence of an equilibrium with two or more bins. The following theorem characterizes the equilibrium with two bins, and forms a basis for equilibria with more bins:

Theorem 2.1: When the source has an exponential distribution with parameter $\lambda$, there exist only non-informative equilibria if and only if $b \leq-\frac{1}{2 \lambda}$. An equilibrium with at least two bins is achievable if and only if $b>-\frac{1}{2 \lambda}$.

Proof Sketch: Consider the two bins $\left[0=m_{0}, m_{1}\right)$ and $\left[m_{1}, m_{2}=\infty\right)$. Then, the centroids of the bins (the decoder actions) are $u_{1}=\frac{1}{\lambda}-\frac{m_{1}}{\mathrm{e}^{\lambda m_{1}}-1}$ and $u_{2}=\frac{1}{\lambda}+m_{1}$. In view of (3), an equilibrium with these two bins exists if and only if

$$
\mathrm{e}^{\lambda m_{1}}\left(\frac{1}{\lambda}+b-\frac{m_{1}}{2}\right)=\frac{1}{\lambda}+b .
$$

Note that in (4), $m_{1}=0$ is always a solution; however, in order to have an equilibrium with two bins, we need a non-zero solution to (4); i.e., $m_{1}>0$. Investigating further gives that the 
equilibrium with two bins can be achieved only if $b>-\frac{1}{2 \lambda}$. In this case, $m_{1}=\frac{1}{\lambda} W_{0}\left(-(2+2 \lambda b) \mathrm{e}^{-(2+2 \lambda b)}\right)+2\left(\frac{1}{\lambda}+b\right)$, where $W_{0}\left(x \mathrm{e}^{x}\right)=x$ for $-1 \leq x<0$ is the principal branch of the Lambert $W$-function.

Contrarily to the negative bias case, the number of bins at the equilibrium is not bounded when the bias is positive. The following theorem investigates the case in which $b>0$ :

Theorem 2.2: When the source has an exponential distribution with parameter $\lambda$, for $b>0$ and any $N \in \mathbb{N}$,

(i) There exists a unique equilibrium with $N$ bins,

(ii) The bin-lengths are monotonically increasing.

Further, since the two statements above hold for any $N \in$ $\mathbb{N}$, there exists no upper bound on the number of bins at an equilibrium.

Proof Sketch: Matching the best responses of the encoder and decoder; i.e., solving (2) and (3) simultaneously, leads to following recursion for bin-lengths:

$$
g\left(l_{k}\right)=\frac{2}{\lambda}+2 b-h\left(l_{k+1}\right), \quad \text { for } k \leq N-2,
$$

with the initial condition $g\left(l_{N-1}\right)=\frac{2}{\lambda}+2 b$. Here, $g\left(l_{k}\right) \triangleq$ $l_{k} \frac{\mathrm{e}^{\lambda l_{k}}}{\mathrm{e}^{\lambda l_{k}-1}}$ and $h\left(l_{k}\right) \triangleq \frac{l_{k}}{\mathrm{e}^{\lambda l_{k}}-1}$. The analysis on this recursion and $g$ and $h$ functions leads to monotonically increasing binlengths. Further, the solution to the recursion in (5) exists for any $N \in \mathbb{N}$.

Theorem 2.2 shows that, when $b>0$, there exists an equilibrium with $N$ bins for any finite $N \in \mathbb{N}$. The following shows the existence of equilibria with infinitely many bins:

Theorem 2.3: For an exponential source and positive bias $b>0$, there exist equilibria with infinitely many bins. In particular, all bins must have a length of $l^{*}$, where $l^{*}$ is the solution to $g\left(l^{*}\right)=\frac{2}{\lambda}+2 b-h\left(l^{*}\right)$.

Proof Sketch: The existence of a fixed point solution to the recursion in (5) proves the statement.

So far, we have shown that at the equilibrium there is an upper bound on the number of bins when $b<0$; i.e., there can exist only finitely many equilibria with finitely many bins. On the other hand, when $b>0$, there is no upper bound on the number of bins at the equilibrium, and even there exist equilibria with infinitely many bins. Therefore, at this point, it is interesting to examine which equilibrium is preferred by the decision makers; i.e., which equilibrium is more informative (has smaller cost).

Theorem 2.4: The most informative equilibrium is reached with the maximum possible number of bins:

(i) for $b<0$, if there are two different equilibria with $K$ and $N$ bins where $N>K$, the equilibrium with $N$ bins is more informative.

(ii) for $b>0$, the equilibria with infinitely many bins are the most informative ones.

Proof Sketch: For any finite $N \in \mathbb{N}$, comparing the decoder costs between an equilibrium with $N$ bins, $N+1$ bins, and infinitely many bins yields the desired result.

Theorem 2.4 implies that, among multiple equilibria, the one with the maximum number of bins must be chosen by the players; i.e., the payoff dominant equilibrium is selected [20].

\section{GAUSSIAN DistribUtion}

Let $M$ be a Gaussian r.v. with mean $\mu$ and variance $\sigma^{2}$; i.e., $M \sim \mathcal{N}\left(\mu, \sigma^{2}\right)$. Let $\phi(m)=\frac{1}{\sqrt{2 \pi}} \mathrm{e}^{-\frac{m^{2}}{2}}$ be the PDF of a standard Gaussian r.v., and let $\Phi(b)=\int_{-\infty}^{b} \phi(m) \mathrm{d} m$ be its cumulative distribution function (CDF). Then, the expectation of a truncated Gaussian r.v. is the following:

Fact 3.1: The mean of a Gaussian r.v. $M \sim \mathcal{N}\left(\mu, \sigma^{2}\right)$ truncated to $[a, b]$ is $\mathbb{E}[M \mid a<M<b]=\mu-\sigma \frac{\phi\left(\frac{b-\mu}{\sigma}\right)-\phi\left(\frac{a-\mu}{\sigma}\right)}{\Phi\left(\frac{b-\mu}{\sigma}\right)-\Phi\left(\frac{a-\mu}{\sigma}\right)}$.

Now we consider an equilibrium with two bins:

Theorem 3.1: When the source has a Gaussian distribution as $M \sim \mathcal{N}\left(\mu, \sigma^{2}\right)$, there always exists an equilibrium with two bins regardless of the value of $b$.

Proof Sketch: Consider the two bins $\left(-\infty=m_{0}, m_{1}\right)$ and $\left[m_{1}, m_{2}=\infty\right)$. The centroids of the bins (the action of the decoder) are $u_{1}=\mu-\sigma \frac{\phi\left(\frac{m_{1}-\mu}{\sigma}\right)}{\Phi\left(\frac{m_{1}-\mu}{\sigma}\right)}$ and $u_{2}=\mu+\sigma \frac{\phi\left(\frac{m_{1}-\mu}{\sigma}\right)}{1-\Phi\left(\frac{m_{1}-\mu}{\sigma}\right)}$. Then, by utilizing (3), an equilibrium with two bins exists if and only if

$$
\begin{aligned}
& m_{1}=\mu+\frac{\sigma}{2}\left(\frac{\phi\left(\frac{m_{1}-\mu}{\sigma}\right)}{1-\Phi\left(\frac{m_{1}-\mu}{\sigma}\right)}-\frac{\phi\left(\frac{m_{1}-\mu}{\sigma}\right)}{\Phi\left(\frac{m_{1}-\mu}{\sigma}\right)}\right)+b \\
& c \triangleq \frac{m_{1}-\mu}{\Rightarrow} 2 c-\frac{\phi(c)}{1-\Phi(c)}+\frac{\phi(c)}{\Phi(c)}=\frac{2 b}{\sigma} .
\end{aligned}
$$

Defining $f(c) \triangleq 2 c-\frac{\phi(c)}{1-\Phi(c)}+\frac{\phi(c)}{\Phi(c)}$ and observing $\lim _{c \rightarrow-\infty} f(c)=-\infty, \lim _{c \rightarrow \infty} f(c)=\infty$, and $f^{\prime}(c)>0$ show that (6) has always a unique solution, which assures that there always exists an equilibrium with two bins regardless of $b$.

Since the PDF of a Gaussian r.v. is symmetrical about its mean $\mu$, and monotonically decreasing in the interval $[\mu, \infty)$, the following can be obtained using a similar reasoning as in Proposition 2.1:

Proposition 3.1: Suppose there is an equilibrium with $N$ bins for a Gaussian source $M \sim \mathcal{N}\left(\mu, \sigma^{2}\right)$. Then,

(i) if $b<0$, bin-lengths are monotonically increasing and the number of bins in $[\mu, \infty)$ is upper bounded,

(ii) if $b>0$, bin-lengths are monotonically decreasing and the number of bins in $(-\infty, \mu]$ is upper bounded.

Proof Sketch: For $b<0$ and any bin in $[\mu, \infty)$, we obtain $u_{k}-m_{k-1}=\mathbb{E}\left[M \mid m_{k-1} \leq M<m_{k}\right]-m_{k-1} \leq \sigma$ by applying an inequality on the upper bound of the Mill's ratio [23]; i.e., $\frac{\phi(c)}{1-\Phi(c)}<\frac{\sqrt{c^{2}+4}+c}{2}$ for $c>0$. Then, a similar approach as in Proposition 2.1 yields the desired result due to the monotonicity of a Gaussian PDF on $[\mu, \infty)$. Similar arguments hold for $b>0$ on $(-\infty, \mu]$.

After showing that there always exists an equilibrium with two bins independent of $b$, we may ask whether there always exists an equilibrium with $N$ bins, or infinitely many bins. The following theorem answers the second part of this question:

Theorem 3.2: For the Gaussian source $M \sim \mathcal{N}\left(\mu, \sigma^{2}\right)$, there exist equilibria with infinitely many bins.

Proof Sketch: The proof requires individual analysis for the positive and the negative $b$ values. For $b>0$, first, it is shown that finite bin-lengths are lower and upper bounded as $2 b \leq l_{k} \leq 2 b+2 \sigma$. Then, since the number of 
bins in $(-\infty, \mu]$ is bounded for $b>0$ by Proposition 3.1, the position of the left-most bin edge is investigated, and by utilizing the bounds on bin-lengths, it is proved that $\mu-\left\lfloor\frac{\sigma}{2 b}\right\rfloor(2 \sigma-2 b)-2 \sigma \leq m_{1} \leq \mu+2 b+\sigma$. Observe that the set $\mathscr{K} \triangleq\left[\mu-\left\lfloor\frac{\sigma}{2 b}\right\rfloor(2 \sigma-2 b)-2 \sigma, \mu+2 b+\sigma\right] \times$ $[2 b, 2 b+2 \sigma] \times[2 b, 2 b+2 \sigma] \times \cdots\left(\right.$ where $\left.\left\{m_{1}, l_{2}, l_{3}, \cdots\right\} \in \mathscr{K}\right)$ is a convex and compact set by Tychonoff's theorem [24], and based on the left-most bin-edge and the bin-lengths, the other bin-edges can be represented by $m_{i}=m_{1}+\sum_{j=2}^{i} l_{j}$. Hence, another convex and compact set $\widehat{\mathscr{K}}$ can be defined such that $\left\{m_{1}, m_{2}, \cdots\right\} \in \widehat{\mathscr{K}}$. Further, at the equilibrium, the best responses of the encoder and the decoder in (2) and (3) can be combined to define a mapping as follows:

$$
\begin{aligned}
\mathbf{m} \triangleq\left[\begin{array}{c}
m_{1} \\
m_{2} \\
\vdots \\
m_{k} \\
\vdots
\end{array}\right] & =\left[\begin{array}{c}
\frac{\mathbb{E}\left[M \mid m_{0}<M<m_{1}\right]+\mathbb{E}\left[M \mid m_{1}<M<m_{2}\right]}{2}+b \\
\frac{\mathbb{E}\left[M \mid m_{1}<M<m_{2}\right]+\mathbb{E}\left[M \mid m_{2}<M<m_{3}\right]}{2}+b \\
\vdots \\
\frac{\mathbb{E}\left[M \mid m_{k-1}<M<m_{k}\right]+\mathbb{E}\left[M \mid m_{k}<M<m_{k+1}\right]}{2}+b
\end{array}\right] \\
& \triangleq \mathscr{T}(\mathbf{m}) .
\end{aligned}
$$

Note that the mapping $\mathscr{T}(\mathbf{m}): \widehat{\mathscr{K}} \rightarrow \widehat{\mathscr{K}}$ is continuous under the point-wise convergence, and hence, under the product topology. Further, since (countably) infinite product of real intervals is a locally convex vector space, $\widehat{\mathscr{K}}$ is a locally convex space. Hence, there exists a fixed point for the mapping $\mathscr{T}$ such that $\mathbf{m}^{*}=\mathscr{T}\left(\mathbf{m}^{*}\right)$ by Tychonoff's fixed-point theorem [24]. This proves the statement.

\section{CONCLUding REMARKS}

In this paper, the Nash equilibrium of cheap talk has been characterized for exponential and Gaussian sources. For exponential sources, it has been shown that the number of bins is bounded for $b<0$, whereas there exist equilibria with infinitely many bins for $b>0$. Further, it has been proved that, as the number of bins increases, the equilibrium cost of the encoder and decoder reduces. For Gaussian sources, there always exists an equilibrium with infinitely many bins.

Future work includes extending the analysis to arbitrary sources with semi-infinite support and two-sided infinite support, and the investigation of upper bounds on the number of bins and the relation between the number of bins and the equilibrium costs of the players (i.e., equilibrium selection problem). Further, the existence and convergence of equilibria; i.e., under what conditions the best responses of the encoder and the decoder match each other, can be analyzed. It is also interesting to analyze the performance loss due to the misalignment between the objective functions in order to obtain comparisons with optimal quantizers.

\section{ACKNOWLEDGMENT}

Some of the results, in particular Prop. 2.1 and Thm. 2.2 build on the project report [25] written by Philippe Furrer, Stephen Kerner and Stanislav Fabricius.

\section{REFERENCES}

[1] V. Crawford and J. Sobel, "Strategic information transmission," Econometrica, vol. 50, pp. 1431-1451, 1982.

[2] S. Sarıtaş, S. Yüksel, and S. Gezici, "Quadratic multi-dimensional signaling games and affine equilibria," IEEE Transactions on Automatic Control, vol. 62, no. 2, pp. 605-619, Feb. 2017.

[3] S. Sarıtaş, S. Yüksel, and S. Gezici, "Dynamic signaling games with quadratic criteria under Nash and Stackelberg equilibria," arXiv preprint arXiv:1704.03816, 2019.

[4] T. Başar and G. Olsder, Dynamic Noncooperative Game Theory. Philadelphia, PA: SIAM Classics in Applied Mathematics, 1999.

[5] F. Farokhi, A. Teixeira, and C. Langbort, "Estimation with strategic sensors," IEEE Transactions on Automatic Control, vol. 62, no. 2, pp. 724-739, Feb. 2017.

[6] E. Akyol, C. Langbort, and T. Başar, "Information-theoretic approach to strategic communication as a hierarchical game," Proceedings of the IEEE, vol. 105, no. 2, pp. 205-218, Feb. 2017.

[7] M. O. Sayın, E. Akyol, and T. Başar, "Hierarchical multi-stage Gaussian signaling games: Strategic communication and control," arXiv preprint arXiv:1609.09448, 2017.

[8] M. L. Treust and T. Tomala, "Information-theoretic limits of strategic communication," arXiv preprint arXiv:1807.05147, 2018.

[9] E. Kamenica and M. Gentzkow, "Bayesian persuasion," American Economic Review, vol. 101, no. 6, pp. 2590-2615, Oct. 2011.

[10] I. Shames, A. Teixeira, H. Sandberg, and K. Johansson, "Agents misbehaving in a network: a vice or a virtue?" IEEE Network, vol. 26, no. 3, pp. 35-40, May 2012.

[11] J. Sobel, "Signaling games," in Encyclopedia of Complexity and Systems Science, R. A. Meyers, Ed. Springer New York, 2009, pp. 8125-8139.

[12] R. M. Gray and D. L. Neuhoff, "Quantization," IEEE Transactions on Information Theory, vol. 44, no. 6, pp. 2325-2383, Oct. 1998.

[13] P. Fleischer, "Sufficient conditions for achieving minimum distortion in a quantizer,' IEEE International Convention Record, Part I, vol. 12, pp. 104-111, 1964.

[14] A. Trushkin, "Sufficient conditions for uniqueness of a locally optimal quantizer for a class of convex error weighting functions," IEEE Transactions on Information Theory, vol. 28, no. 2, pp. 187-198, Mar. 1982.

[15] J. Kieffer, "Uniqueness of locally optimal quantizer for log-concave density and convex error weighting function," IEEE Transactions on Information Theory, vol. 29, no. 1, pp. 42-47, Jan. 1983.

[16] X. Wu, "On convergence of Lloyd's method I," IEEE Transactions on Information Theory, vol. 38, no. 1, pp. 171-174, Jan. 1992.

[17] A. Gyorgy, T. Linder, P. A. Chou, and B. J. Betts, "Do optimal entropyconstrained quantizers have a finite or infinite number of codewords?" IEEE Transactions on Information Theory, vol. 49, no. 11, pp. 30313037, Nov. 2003.

[18] B. Larrousse, O. Beaude, and S. Lasaulce, "Crawford-Sobel meet LloydMax on the grid," in IEEE International Conference on Acoustics, Speech and Signal Processing (ICASSP), May 2014, pp. 6127-6131.

[19] J. S. Banks and J. Sobel, "Equilibrium selection in signaling games," Econometrica, vol. 55, no. 3, pp. 647-661, 1987.

[20] J. Harsanyi and R. Selten, A General Theory of Equilibrium Selection in Games. Cambridge, MA: The MIT Press, 1988.

[21] M. Mitzkewitz, "Equilibrium selection and simple signaling games," 2017, working paper no. 9.

[22] S. Sarıtaş, P. Furrer, S. Gezici, T. Linder, and S. Yüksel, "On the number of bins in equilibria for signaling games," arXiv preprint arXiv:1901.06738, 2019.

[23] Z. W. Birnbaum, "An inequality for Mill's ratio," The Annals of Mathematical Statistics, vol. 13, no. 2, pp. 245-246, June 1942.

[24] A. Granas and J. Dugundji, Fixed Point Theory. Springer-Verlag New York, 2003.

[25] P. Furrer, S. Kerner, S. Fabricius, S. Yüksel, and T. Linder, "Game theory and information, MTHE 493 Technical Report," Apr. 2014. 